\title{
HARMONIC MAPS AND SINGULARITIES OF PERIOD MAPPINGS
}

\author{
JÜRGEN JOST, YI-HU YANG, AND KANG ZUO
}

(Communicated by Lei $\mathrm{Ni}$ )

\begin{abstract}
We use simple methods from harmonic maps to investigate singularities of period mappings at infinity. More precisely, we derive a harmonic map version of Schmid's nilpotent orbit theorem.
\end{abstract}

\section{Constructions, Results, And COnsequences}

Let $\Delta^{*}(\subset \Delta)$ be the punctured disk $\{z \in \mathbb{C}|0<| z \mid<1\}$ with the Poincaré metric

$$
\sqrt{-1} \frac{d z \wedge d \bar{z}}{|z|^{2}(\log |z|)^{2}}
$$

$G / K$ a symmetric space of noncompact type, here, $G$ is a semisimple Lie group and $K$ the corresponding maximal compact subgroup. Suppose that $\rho: \pi_{1}\left(\Delta^{*}\right) \rightarrow G$ is a unipotent representation, i.e. its images are unipotent elements in $G$, and $u: \mathcal{H} \rightarrow G / K$ a $\rho$-equivariant harmonic map with finite energy. Here, $\mathcal{H}$ is the upper half plane which is considered as the universal covering space of $\Delta^{*}$ under the map $w=e^{\sqrt{-1} z}$. The purpose of this note is, by using techniques from harmonic maps, to consider the singularities of $u$ at the infinity of $\mathcal{H}$ (or equivalently, near the puncture of $\left.\Delta^{*}\right)$.

A prototype of the above problem is the degeneration of the period mapping [3, 12. Let $G / H$ be a period domain (more generally a homogeneous complex manifold, where $H$ is the centralizer of a circle subgroup of $G$; special examples are bounded symmetric domains, 4]); it can be considered as a fiber space over a symmetric space $G / K$ of noncompact type with the compact fiber $K / H, p$ : $G / H \rightarrow G / K$, where $K$ is the maximal compact subgroup of $G$ containing $H$. We remark that the $G / K$ is not in general a complex manifold unless it is a bounded symmetric domain. Then, locally, a period mapping is a $\rho$-equivariant map $\Phi$ from the universal covering space $\mathcal{H}$ of $\Delta^{*}$ into the homogeneous complex manifold $G / H$ for a certain unipotent representation $\rho: \pi_{1}\left(\Delta^{*}\right) \rightarrow G$, where $\Phi$ is

1) holomorphic and

2) horizontal with respect to $p: G / H \rightarrow G / K$.

In general, the period mapping is singular at the puncture. This singularity was analyzed in depth in the fundamental paper of Wilfried Schmid [12. The period

Received by the editors December 18, 2013.

2010 Mathematics Subject Classification. Primary 14M27, 58E20.

Key words and phrases. Period mapping, nilpotent orbit, harmonic map.

The first author was partially supported by ERC Advanced Grant FP7-267087.

The second author was partially supported by NSF of China (No. 11171253). 
mapping defined above arises naturally from a Variation of Hodge Structure defined over the rational number field $\mathbb{Q}$ up to a finite lifting.

An observation of $\mathrm{Lu}$ (cf. [10, Theorem 1.1) shows that $p$, restricted to a horizontal slice of $G / H$, is pluriharmonic. So, this, together with the horizontality of $\Phi$, implies that the composition $p \circ \Phi$ is harmonic. On the other hand, $\Phi$ is of finite energy on $\Delta^{*}$ (or a fundamental domain), and so the composition $p \circ \Phi$ is also of finite energy. This can be seen by using two different arguments. One is to observe the asymptotic behavior (cf. e.g. 14]) of the Gauss-Manin connection (or the differential of $\Phi$ ): $\frac{d z}{z} N$ (here $N$ is a nilpotent element in the corresponding Lie algebra; see also the discussion below), which has finite $L^{2}$ norm under the Poincaré metric, and so $\Phi$ has finite energy; obviously, this argument is based on Schmid's theory [12. The other 1$]$ due to Lu and Sun, is that by computing the curvature of the Hodge metric, one can get the finiteness of volume of holomorphic subvarieties under the Hodge metric, in particular $\Phi$ has finite energy (for details, cf. 11, Theorem 5.2 and its proof). This argument depends on the Schwarz lemma and is independent of Schmid's theory.

Return to the above equivariant harmonic maps situation. Without loss of generality, we can restrict ourselves to the case of $G=S L(n, \mathbb{C})$ or one of its semisimple subgroups. Let $\gamma$ be a generator of $\pi_{1}\left(\Delta^{*}\right)$. By the assumption, $\log \rho(\gamma)$ is nilpotent, denoted by $N$. Then, by Jacobson-Morozov's theorem (cf. e.g. [12]), we can obtain a semisimple element $Y$ so that $\{N, Y\}$ can be extended to an $\mathfrak{s l}_{2}$-triple $\left\{N, N^{-}, Y\right\}$. Moreover, such an $\mathfrak{s l}_{2}$-triple is unique up to conjugation in $G[9$.

Now, we can construct a canonical $\rho$-equivariant mapping from the universal covering $\mathcal{H}$ of $\left(\Delta^{*}\right)$ into the symmetric space $S L(n, \mathbb{C}) / S U(n)$ as follows. Let $\mathcal{P}_{n}$ be the set of positive definite Hermitian symmetric matrices of order $n$ with determinant 1. $S L(n, \mathbb{C})$ acts transitively on $\mathcal{P}_{n}$ by

$$
g \circ H=: g H \bar{g}^{t}, H \in \mathcal{P}_{n}, g \in S L(n, \mathbb{C}) .
$$

Obviously, the action has the isotropy subgroup $S U(n)$ at the identity $I_{n}$. Thus, $\mathcal{P}_{n}$ can be identified with the symmetric space of noncompact type $S L(n, \mathbb{C}) / S U(n)$. In particular, under the invariant metric on $\mathcal{P}_{n}$, the geodesics through the identity $I_{n}$ are of the form $\exp (t A), t \in \mathbb{R}$, where $A$ is a Hermitian symmetric matrix. Write $z=r e^{\sqrt{-1} \theta} ;(-\log r, \theta)$ can be considered as the coordinate of $\mathcal{H}$. Now, we can set

$$
h_{0}(z)=\exp \left(\frac{1}{2 \pi} \theta N\right) \circ \exp \left(\left(\frac{1}{2} \log |\log r|\right) Y\right),
$$

which is a $\rho$-equivariant map from $\mathcal{H}$ into the symmetric space $S L(n, \mathbb{C}) / S U(n)$.

Geometrically, such a construction gives an equivariant geodesic embedding of the upper half plane into the symmetric space $S L(n, \mathbb{C}) / S U(n)$. Based on this, we can consider this map as a canonical one. So, as a map from $\mathcal{H}$ into $S L(n, \mathbb{C}) / S U(n), h_{0}$ is harmonic. In the sequel, for sake of convenience, we also consider a $\rho$-equivariant map from $\mathcal{H}$ as a $\rho$-equivariant map from the punctured disk. An easy computation also shows that it is of finite energy (on a fundamental domain w.r.t. the representation $\rho$ ).

\footnotetext{
${ }^{1}$ The second author thanks Zhiqin Lu for informing him of this observation.
} 
In this note, we want to prove the following.

Theorem. Suppose $h: \Delta^{*} \rightarrow S L(n, \mathbb{C}) / S U(n)$ is a $\rho$-equivariant harmonic map with finite energy. Then, $h$ has the same asymptotic behavior as $h_{0}$ near the puncture of $\Delta^{*}$. More precisely, under the invariant metric of $\mathcal{P}_{n}$, the distance function between $h$ and $h_{0}$ is uniformly bounded on $\Delta^{*}$.

Remark. The theorem can be considered as the version for harmonic maps of Schmid's nilpotent orbit theorem.

The representation $\rho$ induces an $n$-dimensional flat complex vector bundle over $\Delta^{*}$ by considering $\rho$ as a representation on $\mathbb{C}^{n}$, denoted by $L_{\rho}$. Then, the map $h_{0}$ (and $h$ ) can be considered as a metric on $L_{\rho}$-harmonic metric (cf. e.g. [13]), which is a natural generalization of the Hodge metric on a variation of Hodge structure. We shall now analyze the asymptotic behavior of $h_{0}$ (and $h$ ) as a metric on $L_{\rho}$. The main issue is the $r$-direction, since the $\theta$-direction is compact (equivariant).

Without loss of generality, we may assume here that the representation is irreducible. This means that the Jordan normal form of $N$ has only one Jordan block. Let $\left\{N, N^{-}, Y\right\}$ be the corresponding $\mathfrak{s l}_{2}$-triple. The semisimple element $Y$ can actually be described as follows. Canonically, $\mathbb{C}^{n}$ has a filtration

$$
0 \subset W_{-(n-1)} \subset W_{-(n-3)} \subset \cdots \subset W_{n-3} \subset W_{n-1}=\mathbb{C}^{n},
$$

with the properties that $N\left(W_{i}\right) \subset W_{i-2}, Y$ preserves each $W_{i}$, and all the quotients $W_{i} / W_{i-2}$ are 1-dimensional. Then the (induced) action of $Y$ on $W_{i} / W_{i-2}$ is multiplication by $i$. Actually, one can also choose a basis

$$
\left\{e_{-(n-1)}, e_{-(n-3)}, \cdots, e_{n-3}, e_{n-1}\right\}
$$

of $\mathbb{C}^{n}$, which is compatible with the above filtration (i.e. $N e_{j}=e_{j-2}$ and $\left\{e_{j}\right\}_{j \leq i}$ generate $\left.W_{i}\right)$ and satisfies $Y e_{i}=i e_{i} . \exp \left(\left(\frac{1}{2} \log |\log r|\right) Y\right)$, under the above basis, can then be written as

$$
\left(\begin{array}{ccccc}
|\log r|^{\frac{-(n-1)}{2}} & 0 & \cdots & 0 & 0 \\
0 & |\log r|^{\frac{-(n-3)}{2}} & \cdots & 0 & 0 \\
\vdots & \vdots & \ddots & \vdots & \vdots \\
0 & 0 & \cdots & |\log r|^{\frac{n-3}{2}} & 0 \\
0 & 0 & \cdots & 0 & |\log r|^{\frac{n-1}{2}}
\end{array}\right) .
$$

On the other hand, by using the invariant metric of $\mathcal{P}_{n}$, a simple computation also shows that the energy of $h_{0}$ is finite, namely,

$$
E\left(h_{0}\right)=\int_{\Delta^{*}}\left|h_{0}^{-1} d h_{0}\right|^{2} * 1 \leq C \int_{0}^{\alpha}|\log r|^{-2} r^{-1} d r+\infty .
$$

From the above argument, we can now see the asymptotic behavior of the norms near the puncture of flat sections of $L_{\rho}$ under the metric $h_{0}$ (and hence $h$ ). For $p \in \Delta^{*}$, the fiber $\left(L_{\rho}\right)_{p}$ canonically has a weight filtration $\left\{W_{l}\right\}_{l=-k}^{k}(k$ is the weight of $N$ ) arising from $N$ and satisfying $N\left(W_{l}\right) \subset W_{l-2}$; this filtration is moreover invariant w.r.t. the flat connection $D$ of $L_{\rho}$ and hence determines a filtration of $L_{\rho}$ by some local subsystems, denoted by $\mathbf{W}_{l},-k \leq l \leq k$. We remark that $\left\{W_{l}\right\}_{l=-k}^{k}$ can be decomposed into the direct sum of some subfiltrations, each of which corresponds to a unique Jordan block of $N$ and is of the form in (2) if the number of the Jordan blocks of $N$ is $>1$. By the construction of $h_{0}$ and (3), a flat 
section $v$ of $\mathbf{W}_{l}$, if not lying in $\mathbf{W}_{l-1}$, has the following norm estimate 2 under $h_{0}$ (and hence $h$ ):

$$
\|v\|^{2} \sim|\log r|^{l}
$$

on any ray from the puncture of $\Delta^{*}$. This is just Schmid's norm estimate for Hodge metrics 12 .

The basic idea of this note was already utilized in our joint paper [7, where we discussed the cohomologies of harmonic bundles over noncompact curves. So, this note can also be considered as an appendix of 7 .

\section{Proof of Theorem}

The proof of the Theorem utilizes some simple and well-known facts about harmonic maps; see e.g. [5] as a reference. From the point of view of geometric analysis, the key point is that the target space $G / K$ as a symmetric space of noncompact type has nonpositive sectional curvature. Let $\Delta_{i}^{*}\left(\subset \Delta^{*}\right)$ be the punctured disk with the radius $\frac{1}{i}$. By minimizing the energy among all $\rho$-equivariant maps with prescribed values on the boundary of $\Delta^{*} \backslash \Delta_{i}^{*}$ given by the values of $h$ on that boundary, we obtain a $\rho$-equivariant harmonic map $h_{i}$ on $\Delta^{*} \backslash \Delta_{i}^{*}$ with

$$
\begin{array}{rc}
\left.h_{i}\right|_{\partial \Delta} & =\left.h_{0}\right|_{\partial \Delta}, \\
\left.h_{i}\right|_{\partial \Delta_{i}} & =\left.h\right|_{\partial \Delta_{i}}, \\
E\left(h_{i}, \Delta^{*} \backslash \Delta_{i}^{*}\right) & \leq E\left(h, \Delta^{*} \backslash \Delta_{i}^{*}\right)+C \leq E\left(h, \Delta^{*}\right)+C<+\infty,
\end{array}
$$

where $C$ is some nonnegative constant independent of $i$. (That $C$ need not be 0 stems from the fact that on $\partial \Delta$, we impose the boundary values of $h_{0}$ and not those of $h$.) On the other hand, by a standard computation, the function

$$
\left(\operatorname{dist}_{\mathcal{P}_{n}}\left(h_{i}, h\right)\right)^{2}
$$

is subharmonic on $\Delta^{*} \backslash \Delta_{i}^{*}$. So, by the maximum principle, we have

$$
\left.\operatorname{dist}_{\mathcal{P}_{n}}\left(h_{i}, h\right)\right|_{\Delta^{*} \backslash \Delta_{i}^{*}} \leq \max _{\partial \Delta} \operatorname{dist}_{\mathcal{P}_{n}}\left(h, h_{0}\right) .
$$

Thus, (5) and (6) together with standard estimates imply that $h_{i}$ (possibly after selecting a subsequence) converges uniformly on (any compact subset of) $\Delta^{*}$ to some harmonic limit $\tilde{h}$ that satisfies

$$
\begin{aligned}
\left.\tilde{h}\right|_{\partial \Delta} & =\left.h_{0}\right|_{\partial \Delta}, \\
E\left(\tilde{h}, \Delta^{*}\right) & <+\infty .
\end{aligned}
$$

All together, we get a $\rho$-equivariant harmonic map $\tilde{h}: \Delta^{*} \rightarrow S L(n, \mathbb{C}) / S U(n)$ with

$$
\begin{aligned}
& E\left(\tilde{h}, \Delta^{*}\right)+\infty, \\
& \operatorname{dist}_{\mathcal{P}_{n}}(h, \tilde{h}) \leq \max _{\partial \Delta} \operatorname{dist}_{\mathcal{P}_{n}}\left(h, h_{0}\right), \text { on } \Delta^{*}, \\
& \left.\tilde{h}\right|_{\partial \Delta}=\left.h_{0}\right|_{\partial \Delta} .
\end{aligned}
$$

By $(10), h$ and $\tilde{h}$ have the same asymptotic behavior near the puncture. We now want to show that actually $\tilde{h} \equiv h_{0}$ on $\Delta^{*}$, and hence the Theorem is obtained.

\footnotetext{
${ }^{2}$ Here, we use the notation $\sim$ to mean "is within a bounded multiple of".
} 
Consider the square of the distance function between $\tilde{h}$ and $h_{0}$,

$$
\left(\operatorname{dist}_{\mathcal{P}_{n}}\left(\tilde{h}, h_{0}\right)\right)^{2},
$$

which is defined on $\Delta^{*}$ and subharmonic because of the nonpositive sectional curvature of $G / K$. Furthermore, $\left(\operatorname{dist}_{\mathcal{P}_{n}}\left(\tilde{h}, h_{0}\right)\right)^{2}$ is of finite energy. Actually, the energy finiteness of $\tilde{h}$ and $h_{0}$, by Cheng's gradient estimate for harmonic maps, implies that they have bounded energy density under the Poincaré metric

$$
\sqrt{-1} \frac{d z \wedge d \bar{z}}{|z|^{2}(\log |z|)^{2}}
$$

Consequently, $\operatorname{dist}_{\mathcal{P}_{n}}\left(\tilde{h}, h_{0}\right) \leq C \log |\log r|$, for some constant $C$. This, together with the energy finiteness of $\tilde{h}$ and $h_{0}$, implies that $\left(\operatorname{dist}_{\mathcal{P}_{n}}\left(\tilde{h}, h_{0}\right)\right)^{2}$ is of finite energy. The theorem now follows from the following elementary lemma; for completeness, we reduce it to a standard result.

Lemma 1. Let $\Delta^{*}$ be the punctured disk with the Poincaré metric. Then any finite energy nonnegative subharmonic function $w$ that vanishes on the exterior boundary vanishes identically in $\Delta^{*}$.

Proof. Because of the conformal invariance of the Dirichlet integral in the 2-dimensional case, neither the finiteness of the energy nor the subharmonicity depend on the metrics on the punctured disk (as long as it is conformally equivalent to the hyperbolic one). So, instead of the complete hyperbolic metric, we may as well use the incomplete Euclidean one on $\Delta^{*}$. Consider the following sequence of harmonic functions

$$
\begin{cases}u_{i} & \text { harmonic function on } \Delta^{*} \backslash \Delta_{i}^{*}, \\ \left.u_{i}\right|_{\partial \Delta}=\left.w\right|_{\partial \Delta}=0, \\ \left.u_{i}\right|_{\partial \Delta_{i}}=\left.w\right|_{\partial \Delta_{i}} .\end{cases}
$$

The maximum principle implies $u_{i} \geq w$ on $\Delta^{*} \backslash \Delta_{i}^{*}$ and

$$
E\left(u_{i} ; \Delta^{*} \backslash \Delta_{i}^{*}\right) \leq E\left(w ; \Delta^{*} \backslash \Delta_{i}^{*}\right) \leq E(w) .
$$

The standard elliptic estimate then implies that $u_{i}$ converges uniformly on (any compact subset of) $\Delta^{*}$ to a harmonic function $u$, which satisfies $u \geq w,\left.u\right|_{\partial \Delta}=0$, and $E(u) \leq E(w)$. Then, $u \equiv 0$, and hence $w \equiv 0$, follows from the standard removability of isolated singularities of finite energy harmonic functions (see e.g. Corollary 11.2.2. in [6]), and the maximum principle.

\section{ACKNOWLEDGEMENT}

The second author thanks Zhiqin Lu at Irvine for his interest and comments.

\section{REFERENCES}

[1] Eduardo Cattani, Aroldo Kaplan, and Wilfried Schmid, Degeneration of Hodge structures, Ann. of Math. (2) 123 (1986), no. 3, 457-535, DOI 10.2307/1971333. MR840721 (88a:32029)

[2] Maurizio Cornalba and Phillip Griffiths, Analytic cycles and vector bundles on non-compact algebraic varieties, Invent. Math. 28 (1975), 1-106. MR0367263 (51 \#3505)

[3] Phillip Griffiths (ed.), Topics in transcendental algebraic geometry, Annals of Mathematics Studies, vol. 106, Princeton University Press, Princeton, NJ, 1984. MR756842 (86b:14004)

[4] Phillip Griffiths and Wilfried Schmid, Locally homogeneous complex manifolds, Acta Math. 123 (1969), 253-302. MR0259958 (41 \#4587)

[5] Jürgen Jost, Riemannian geometry and geometric analysis, 6th ed., Universitext, Springer, Heidelberg, 2011. MR2829653 
[6] Jürgen Jost, Partial differential equations, 3rd ed., Graduate Texts in Mathematics, vol. 214, Springer, New York, 2013. MR3012036

[7] Jürgen Jost, Yi-Hu Yang, and Kang Zuo, Cohomologies of unipotent harmonic bundles over noncompact curves, J. Reine Angew. Math. 609 (2007), 137-159, DOI 10.1515/CRELLE.2007.062. MR2350782 (2009e:32021)

[8] J. Jost, Y.-H. Yang, and K. Zuo, Harmonic metrics on unipotent bundles over quasi-compact Kähler manifolds. Preprint.

[9] Bertram Kostant, The principal three-dimensional subgroup and the Betti numbers of a complex simple Lie group, Amer. J. Math. 81 (1959), 973-1032. MR0114875 (22 \#5693)

[10] Z. Lu, On the geometry of classifying spaces and horizontal slices. American Journal of Mathematics 121(1999), 177-198. MR1705002 (2000j:14057)

[11] Zhiqin Lu and Xiaofeng Sun, Weil-Petersson geometry on moduli space of polarized Calabi-Yau manifolds, J. Inst. Math. Jussieu 3 (2004), no. 2, 185-229, DOI 10.1017/S1474748004000076. MR2055709 (2005j:32012)

[12] Wilfried Schmid, Variation of Hodge structure: the singularities of the period mapping, Invent. Math. 22 (1973), 211-319. MR0382272 (52 \#3157)

[13] Carlos T. Simpson, Harmonic bundles on noncompact curves, J. Amer. Math. Soc. 3 (1990), no. 3, 713-770, DOI 10.2307/1990935. MR.1040197 (91h:58029)

[14] Steven Zucker, Hodge theory with degenerating coefficients. L $L_{2}$ cohomology in the Poincaré metric, Ann. of Math. (2) 109 (1979), no. 3, 415-476, DOI 10.2307/1971221. MR.534758 (81a:14002)

Max-Planck Institute for Mathematics in the Sciences, 04103 Leipzig, Germany

E-mail address: jjost@mis.mpg.de

Department of Mathematics, Shanghai Jiao Tong University, Shanghai, 200240, People's Republic of China

E-mail address: yangyihu@sjtu.edu.cn

Department of Mathematics, Mainz University, 55099 Mainz, Germany

E-mail address: zuok@uni-mainz.de 